\title{
Construcción y validación inicial de un instrumento para medir el estilo de vida en pacientes con diabetes mellitus tipo 2
}

Juan Manuel López-Carmona, M en Invest Clín, ${ }^{(1)}$ C uauhtémoc Raúl A riza-Andraca, MC, ${ }^{(2)}$

José Raymundo Rodríguez-Moctezuma, MC, M en Invest Clín, ${ }^{(3)}$ Catarina Munguía-Miranda, M en C. ${ }^{(4)}$

\begin{abstract}
López-Carmona JM,Ariza-Andraca CR, Rodríguez-Moctezuma JR, Munguía-Miranda C. Construcción y validación inicial

de un instrumento para medir el estilo de vida en pacientes con diabetes mellitus tipo 2. Salud Publica Mex 2003;45:259-268.

El texto completo en inglés de este artículo está
\end{abstract} disponible en: http://www.insp.mx/salud/index.html

\section{Resumen}

Objetivo. Construir y validar un instrumento específico para medir el estilo de vida en los pacientes con diabetes mellitus tipo 2. Material y métodos. Entre marzo de 2001 y abril de 2002 se hizo un estudio observacional, longitudinal y prospectivo en unidades de medicina familiar de la delegación Estado de México 0 riente, del Instituto Mexicano del Seguro Social. Se diseñó un instrumento de autoadministración, denominado instrumento para medir el estilo de vida en diabéticos, el cual fue sometido a revisión por un panel multidisciplinario de expertos para determinar su validez ló gica y de contenido. Se aplicó el instrumento en dos días diferentes a 412 sujetos adultos con diabetes mellitus. Fueron depurados los ítems considerando la frecuencia de selección de sus opciones de respuesta, su correlación ítem-total y su carga significativa en los dominios durante el análisis factorial. Resultados. Completaron el estudio 389 (94.7\%) sujetos. Los coeficientes de correlación intra-clase para la validez lógica y de contenido fueron de 0.91 y 0.95 , respectivamente. Después de la depuración de ítems se obtuvo un instrumento conformado por 25 preguntas cerradas, distribuidas en siete dominios: nutrición, actividad

\author{
López-Carmona JM,Ariza-Andraca CR, \\ Rodríguez-Moctezuma JR, Munguía-Miranda C. \\ Development and initial validation \\ of an instrument to measure lifestyle \\ of type 2 diabetes mellitus patients. \\ Salud Publica Mex 2003;45:259-268. \\ The English version of this paper \\ is available at: http://www.insp.mx/salud/index.html
}

\begin{abstract}
A bstract
O bjective.To develop and validate a specific instrument to measure lifestyle of type 2 diabetes mellitus (DM2) patients. Material and Methods. A lo ngitudinal, observational, prospective study was conducted between March 2001 and A pril 2002 at family medicine healthcare units of the Instituto Mexicano del Seguro Social (Mexican Institute of Social Security (MISS)) in Mexico State's East District. A self-administered instrument (instrument to measure diabetic lifestyles, IM EVID) was designed to measure the lifestyles of diabetic patients. It was submitted for review by a multidisciplinary group of experts who assessed its logical and content validity to measure the lifestyle in DM2 patients. The instrument was administered on two different days to 412 adult subjects with DM2. Some items were excluded on the basis of the frequency with which their answer choices were selected, as well as the item-total correlation, and the item's significant loads in several domains in factorial analysis. Results. The sample population consisted of 389 (94.7 $\%$ response) subjects who completed the study. Intraclass correlation coefficients for logical and content validity were 0.91 and 0.95 respectively. After depuration of items, the
\end{abstract}

Trabajo realizado gracias al apoyo financiero otorgado por la Coordinación de Investigación en Salud del Instituto Mexicano del Seguro Social a través del Fondo para el Fomento a la Investigación con número FP-2001/200.

Trabajo de tesis del primer autor para obtener el grado de Maestro en Investigación Clínica, por la Universidad Autónoma del Estado de México.

(1) Unidad de Medicina Familiar N 0.91 Coacalco. Delegación Estado de México O riente, Instituto Mexicano del Seguro Social (IMSS), México.

(2) Centro Médico N acional "La Raza", D elegación 2 N oreste, IMSS, México, D F, México.

(3) Hospital G eneral Regional N 0.72."Lic.Vicente Santos Guajardo", D elegación Estado de México 0 riente, IMSS, México.

(4) Delegación Estado de México 0 riente, IMSS, México.

Fecha de recibido: 22 de agosto de 2002 - Fecha de aprobado: 18 de marzo de 2003

Solicitud de sobretiros: Juan Manuel López Carmona. Hospital General Regional N 0. 72. Módulo de Investigación 20 Piso, Avenida Filiberto Gómez sin número esquina Gustavo Baz,Tlalnepantla 54000, Estado de México, México.

Correo electrónico:jmlopcar@ prodigy.net.mx 
física, consumo de tabaco, consumo de alcohol, información sobre diabetes, emociones y adherencia terapéutica. El $\alpha$ de $C$ ronbach para la calificación total fue de 0.81 y el coeficiente de correlación test-retest de 0.84 . Conclusiones. El instrumento para medir el estilo de vida en diabéticos es el primer cuestionario específico para sujetos con diabetes mellitus tipo 2 y tiene validez aparente, validez de contenido y buen nivel de consistencia. El texto completo en inglés de este artículo está disponible en:http://www.insp.mx/ salud/index.html

Palabras clave: diabetes mellitus no insulino dependiente; estilo de vida; cuestionario; validación; México instrument had 25 closed items grouped in 7 domains: nutrition, physical activity, to bacco consumption, alcohol consumption, information on diabetes, emotions, and therapeutic adherence. The global rating of the questionnaire had a Cronbach's alpha of 0.81 and a test-retest correlation coefficient of 0.84 . Conclusions. The specific questionnaire IMEVID is the first to measure the lifestyle in subjects with D M2. It has logical validity, content validity, and a go od level of consistency. The English version of this paper is available at: http://www.insp.mx/salud/index.html

Key words: lifestyle; diabetes mellitus, non-insulin-dependent questionnaires; validation; Mexico a diabetes mellitus tipo 2 (DM2) es consecuencia L de la interacción de factores genéticos y ambientales entre los que el estilo de vida juega un papel fundamental. El conocimiento del estilo de vida y su medición es una prioridad para los médicos que atienden a pacientes con DM2, pues algunos de sus componentes como tipo de alimentación, ${ }^{1,2}$ actividad física, ${ }^{3}$ presencia de obesidad, ${ }^{4}$ consumo de alcohol ${ }^{5}$ y consumo de tabaco, ${ }^{6}$ entre otros, se han asociado a la ocurrencia, curso clínico y control de la diabetes. La modificación de estos factores puede retrasar o prevenir la aparición de la diabetes o cambiar su historia natural. 7,8

La Organización Mundial de la Salud considera al estilo de vida como la manera general de vivir que se basa en la interacción entre las condiciones de vida y los patrones individuales de conducta, los cuales están determinados por factores socioculturales y por las características personales de los individuos. De esta manera, ha propuesto la siguiente definición: "Los estilos de vida son patrones de conducta que han sido elegidos de las alternativas disponibles para la gente, de acuerdo a su capacidad para elegir y a sus circunstancias socioeconómicas". 9 .

Entre los dominios que integran el estilo de vida se han incluido conductas y preferencias relacionadas con el tipo de alimentación, actividad física, consumo de alcohol, tabaco u otras drogas, responsabilidad para la salud, actividades recreativas, relaciones interpersonales, prácticas sexuales, actividades laborales y patrones de consumo. ${ }^{10-12}$

Los pocos instrumentos disponibles para medir el estilo de vida son de tipo genérico, es decir, construidos para aplicarse a población general y no a personas con enfermedades específicas. Dos de los cuestionarios más conocidos de este tipo son el FANTASTIC ${ }^{10} \mathrm{y}$ el Health-Promoting Lifestyle Profile (HPLP). ${ }^{12}$ Estos instrumentos han sido traducidos al idioma español pero su utilización clínica o en investigación ha sido escasa. El instrumento FANTASTIC no ha demostrado su utilidad real en la práctica clínica como una herramienta que permita identificar los estilos de vida "poco saludables" en pacientes con enfermedades específicas como la hipertensión arterial ${ }^{13}$ o la diabetes mellitus. ${ }^{14}$

Se ha sugerido que los instrumentos específicos para medir estados de salud pueden tener mejor rendimiento que los genéricos, debido a que están enfocados a los dominios más relevantes de la enfermedad o condición en estudio y a las características de los pacientes, lo cual hace que sean más apropiados para utilizarse en ensayos clínicos en los que se evalúan tratamientos $\mathrm{u}$ otras intervenciones específicas. ${ }^{15-17}$

No existen instrumentos específicos diseñados para medir los estilos de vida en los sujetos con diabetes. Un instrumento construido para este fin puede facilitar la identificación y medición de los componentes del estilo de vida que se relacionan con el curso clínico de la diabetes, el control metabólico y el pronóstico. La identificación de estos componentes es fundamental para proporcionar consejería individual o familiar de manera adecuada y oportuna, así como para proponer la integración de los pacientes a programas grupales de intervención, dirigidos a fomentar estilos de vida "favorables" para lograr un mejor control metabólico. La aplicación seriada del instrumento podría ser de utilidad también para evaluar el efecto de estas maniobras de intervención.

El objetivo de este estudio fue construir y validar un instrumento específico para medir el estilo de vida en los pacientes con DM2.

\section{Material y métodos}

Entre marzo de 2001 y abril de 2002 se realizó un estudio observacional, longitudinal y prospectivo en las 
Unidades de Medicina Familiar (UMF) 62, 64, 91, 184, 185 y 186 de la Delegación Estado de México Oriente, del Instituto Mexicano del Seguro Social, ubicadas en el área metropolitana de la Ciudad de México. El protocolo fue aprobado por un comité local de investigación.

\section{Construcción del instrumento}

Después de revisar la literatura sobre la metodología para la construcción y validación de cuestionarios, ${ }^{18-22}$ conceptos y definición del estilo de vida, dominios que lo comprenden, ${ }^{9-12}$ y su asociación con la diabetes, se elaboró la primera versión de un instrumento de autoadministración para medir el estilo de vida en pacientes con DM2. El cuestionario estaba constituido por 42 preguntas cerradas, con tres opciones de respuesta cada una, agrupadas en 11 dominios.

La validez lógica y de contenido se evaluó mediante consenso de expertos, ${ }^{18}$ por un panel multidisciplinario de 16 profesionales de la salud conformado por médicos internistas, endocrinólogos, psicólogos clínicos, médicos familiares, nutricionistas e investigadores clínicos, quienes evaluaron el instrumento de manera independiente y cegada, todos ellos con más de cinco años de experiencia en la atención de pacientes con diabetes.

Esta primera versión del instrumento también se aplicó a un grupo piloto de 52 pacientes con DM2 para evaluar el nivel de comprensión, legibilidad y reproducibilidad.

Una vez concluida la revisión del instrumento por el panel de expertos y efectuada la prueba piloto, se hicieron modificaciones en la redacción de ocho ítems, se eliminaron tres y se agregaron dos más, se reagruparon los dominios eliminándose dos de ellos. El instrumento final para su aplicación a la muestra de sujetos contenía 41 ítems agrupados en nueve dominios: nutrición, actividad física, consumo de tabaco, consumo de alcohol, relación con la familia y amistades, información sobre diabetes, satisfacción laboral, emociones y adherencia al tratamiento.

\section{Sujetos}

Se estudió una muestra no probabilística de 412 pacientes con diabetes tipo 2, adultos, de ambos géneros, que supieran leer y escribir, elegidos de entre quienes acuden a recibir atención médica para su diabetes en las UMF participantes. No se incluyeron pacientes diabéticos tipo 1, ni diabéticas embarazadas. El tamaño de la muestra se calculó considerando la inclusión de
10 sujetos por cada ítem del instrumento, número necesario para efectuar análisis multivariante de factores.

\section{Administración del cuestionario}

Los participantes contestaron el cuestionario de manera auto-administrada en una aula de las UMF participantes y regresaron entre 15 y 30 días después para contestar el instrumento por segunda ocasión, considerando este lapso lo suficientemente prolongado como para no recordar las respuestas dadas en la primera administración y lo suficientemente corto como para presentar cambios importantes en el estilo de vida. Lapsos similares se han utilizado para evaluar la consistencia test-retest del FANTASTIC ${ }^{13,14,22}$ y del HPLP. ${ }^{23}$

Se manejó una hoja por separado, con preguntas sobre variables sociodemográficas.

\section{Depuración de los ítems}

Una vez que el instrumento fue aplicado a la muestra de sujetos, se depuraron los ítems teniendo en cuenta varios criterios. En primer lugar, se eliminaron los ítems cuya frecuencia en alguna de las alternativas de respuesta fuera inferior a $5 \%$ o superior a $95 \%$, por su bajo poder de discriminación. Los ítems cuya correlación entre su calificación con el total de su dominio y con el total del cuestionario fue menor a 0.20 también fueron eliminados por su escasa aportación a la calificación total. Se realizó análisis exploratorio de componentes principales, utilizando los autovalores mayores a $1 \mathrm{y}$, posteriormente, análisis factorial con rotación ortogonal y oblicua, ${ }^{24}$ métodos que permiten una separación más clara de los factores, al maximizar las correlaciones entre los ítems y sus dominios. Los ítems que presentaron cargas significativas en varios dominios fueron eliminados.

\section{A nálisis de los datos}

La estadística descriptiva se presenta por medio de proporciones o razones para las variables categóricas, medidas de tendencia central y de dispersión para las variables cuantitativas, anotadas como medias, y desviación estándar si los datos presentan una distribución normal, o como medianas si se muestran libres de distribución.

El grado de acuerdo entre los panelistas para establecer la validez aparente y la de contenido del instrumento se estableció mediante el coeficiente de correlación intra-clase. La consistencia interna del cuestionario se determinó mediante el coeficiente de con- 
fiabilidad alfa de Cronbach, ${ }^{19}$ y la consistencia externa test-retest fue evaluada mediante coeficiente de correlación de Spearman.

Se buscaron diferencias en las calificaciones según el género, tiempo desde el diagnóstico de la DM2 (punto de corte en cinco años) y edad (punto de corte en 60 años), utilizando la prueba U de Mann Withney.

Se utilizó el paquete estadístico SPSS 9 para Windows.

\section{Resultados}

Participaron un total de 412 pacientes, de los cuales $389(94.4 \%)$ respondieron completo el cuestionario y fueron incluidos en el análisis. Rechazaron la invitación a participar ocho pacientes.

La edad de las mujeres estaba comprendida entre 19 y 80 años, media \pm desviación estándar $(D E)=$ $54.8 \pm 10.8$, y la de los hombres entre 19 y 78 años, media $\pm D E=56.0 \pm 11.8$. El tiempo desde el diagnóstico de la DM2 en el género femenino tuvo un mínimo de 1 mes y un máximo de 30 años, media $\pm D E=6.7 \pm 6.0$, en tanto que en el género masculino el mínimo fue de 1 mes y el máximo de 33 años, media $\pm D E=7.1 \pm 8.8$ años. Las características demográficas de la muestra se presentan en el cuadro I.

El tiempo necesario para contestar el cuestionario fue en promedio de 17 minutos (amplitud de 7 a 25 minutos). El intervalo promedio test-retest fue de 18 días (amplitud de 15 a 30 días).

\section{Validez lógica y de contenido}

El grado de acuerdo entre los 16 panelistas, en relación con las preguntas dirigidas a establecer la validez lógica y de contenido, tuvieron un coeficiente de correlación intra-clase de 0.91 (IC 95\% 0.84-0.96) y 0.95 (IC 95\% 0.90-0.98), respectivamente, durante la primera ronda, por lo que no se consideró necesario repetirla.

\section{Depuración de ítems y consistencia interna}

El análisis exploratorio del instrumento inicial (41 ítems) identificó 10 componentes principales con autovalores mayores a 1, que explicaban $58.6 \%$ de la varianza de la calificación total. Después de las rotaciones ortogonal y oblicua se eliminaron algunos ítems.

Nutrición. Estaba conformado por 14 ítems, con un valor $\alpha$ de Cronbach de 0.68 . Se eliminaron cinco de ellos por no alcanzar $5 \%$ en una de sus opciones de respuesta o no lograr correlación ítem-total igual o mayor a 0.20. Este dominio quedó finalmente con nueve ítems y una

\section{Cuadro I \\ Características generales de la muestra. MÉxico, 2001-2002}

No. $\quad \%$

Sexo

\begin{tabular}{lll} 
Mujeres & 269 & 69.2 \\
\hline Hombres & 120 & 30.8
\end{tabular}

Edad (años)

\begin{tabular}{lrr}
$<40$ & 32 & 8.2 \\
\hline 40 a 59 & 206 & 53.0 \\
\hline 60 o más & 151 & 38.8
\end{tabular}

0 cupación

\begin{tabular}{lrr} 
Labores del hogar & 245 & 63.0 \\
\hline Trabajador en activo & 102 & 26.2 \\
\hline Pensionado o jubilado & 36 & 9.3 \\
\hline Desempleado & 6 & 1.5
\end{tabular}

Estado civil

\begin{tabular}{lrr} 
Solteros & 23 & 5.9 \\
\hline Casados & 276 & 71.0 \\
\hline Viudos & 65 & 16.7 \\
\hline Divorciados & 11 & 2.8 \\
\hline Unión libre & 14 & 3.6
\end{tabular}

Escolaridad (años)

\begin{tabular}{lrr} 
Hasta 6 & 275 & 70.7 \\
\hline 7 a 9 & 65 & 16.7 \\
\hline 10 a 12 & 36 & 9.2 \\
\hline 13 o más & 10 & 2.6 \\
\hline Sin dato & 3 & 0.8
\end{tabular}

Tiempo de evolución (años)

\begin{tabular}{lrr}
$\leq 5$ & 205 & 52.7 \\
\hline 6 a 10 & 83 & 21.3 \\
\hline$>10$ & 101 & 26.0
\end{tabular}

Tratamiento

\begin{tabular}{lrr} 
Dieta solamente & 47 & 12.1 \\
\hline Antidiabéticos orales & 287 & 73.7 \\
\hline Insulina & 35 & 9.0 \\
\hline Antidiabéticos orales + insulina & 10 & 2.6 \\
\hline Sin dato & 10 & 2.6
\end{tabular}

$n=389$

$\alpha$ de Cronbach de 0.61 . Se identificaron dos componentes principales que explicaron $51.9 \%$ de la varianza. Actividad física. Contaba con cuatro ítems, $\alpha$ de Cronbach de 0.53 . Al eliminar un ítem que tuvo correlación salud pública de méxico / vol.45, no.4, julio-agosto de 2003 
ítem-total menor de 0.20, quedó con tres ítems y $\alpha$ de 0.51 . Se identificó sólo un componente que explica $50.8 \%$ de la varianza.

Consumo de tabaco. Contaba con dos ítems, $\alpha$ de Cronbach de 0.96 y no requirió modificaciones. Tiene un solo componente principal que explica $96 \%$ de la varianza.

Consumo de alcohol. No fue modificado, cuenta con dos ítems, $\alpha$ de Cronbach de 0.90 . Se identificó un componente principal que explica $91.4 \%$ de la varianza.

Relación con la familia y amistades. Contaba inicialmente con tres ítems, uno de los cuales tuvo correlación ítemtotal menor de 0.20 , y los dos restantes tuvieron cargas factoriales significativas en diferentes dominios, por lo cual se decidió eliminar el dominio completo.

Información sobre diabetes. Contaba con tres ítems, $\alpha$ de Cronbach de 0.65 . El análisis factorial indicó que uno de sus ítems correspondía al dominio de adherencia terapéutica por lo cual fue reubicado, quedando finalmente con dos ítems, $\alpha$ de Cronbach de 0.62 y un solo componente principal que explica $72.6 \%$ de la varianza.

Satisfacción laboral. Inicialmente tenía cuatro ítems, $\alpha$ de Cronbach de 0.50. Dos de los ítems no alcanzaron más de $5 \%$ de respuesta en una de sus opciones y dos más no tuvieron correlación ítem-total igual o mayor a 0.20 , por lo que se eliminó el dominio completo.

Emociones. Este dominio, formado inicialmente por seis ítems con un valor $\alpha$ de 0.83 , durante el análisis factorial, perdió tres de ellos pues presentaron cargas significativas en diferentes dominios, quedando finalmente conformado por tres ítems, $\alpha$ de Cronbach de 0.73 y un solo componente que explica $64.9 \%$ de la varianza. Adherencia terapéutica. Contaba con tres ítems, $\alpha$ de Cronbach de 0.65 . En el análisis factorial se identificó un ítem que pertenecía a este dominio y que había sido ubicado originalmente en el dominio Información sobre diabetes. Finalmente quedó con cuatro ítems, y una $\alpha$ de Cronbach de 0.73 . Presentó un componente principal que explica $56.5 \%$ de la varianza.

En resumen, el instrumento final quedó constituido por 25 ítems, agrupados en siete dominios: nutrición, actividad física, consumo de tabaco, consumo de alcohol, información sobre diabetes, emociones y adherencia terapéutica (anexo 1).

El análisis del instrumento final (25 ítems) mostró ocho componentes principales con autovalores mayores a 1, varianzas explicadas decrecientes desde $19.2 \%$ para el primero, hasta $4.1 \%$ en el último, con un total acumulado de $60.4 \%$. Las rotaciones ortogonal y oblicua, durante el análisis factorial confirmatorio, corroboraron la presencia de ocho factores, conceptual- mente idénticos, con cargas de los ítems $>$ de 0.40 para un solo factor. Dos factores correspondieron al dominio nutrición (uno relacionado con el tipo y otro con la cantidad de alimentos) y uno para cada uno de los dominios restantes.

\section{Consistencia interna}

Los valores alfa de Cronbach para las calificaciones por dominio y total de los instrumentos inicial y final se muestran en el cuadro II.

\section{Consistencia externa}

De los 389 sujetos incluidos en el análisis, contestaron el retest 330 (84.8\%). Los coeficientes de correlación testretest se muestran en el cuadro III.

\section{Escala de calificaciones del instrumento}

Se asignaron calificaciones de 0, 2 y 4 a las tres opciones de respuesta, y correspondió al valor más alto para la conducta deseable, lográndose así una escala de 0 a 100 puntos para todo el instrumento.

La mediana de las calificaciones por género, tiempo de evolución y edad, según los puntos de corte seleccionados, se presentan en el cuadro IV.

\section{A nálisis por género}

El análisis por género muestra que las mujeres calificaron mejor en los dominios nutrición, consumo de

\section{Cuadro II \\ Valores $\alpha$ de Cronbach, Por dominio y total del Instrumento para Medir el Estilo de Vida en Diabéticos. México, 2001-2002}

\begin{tabular}{|c|c|c|c|c|}
\hline \multirow[b]{2}{*}{ Dominios } & \multicolumn{2}{|c|}{ Cuestionario inicial } & \multicolumn{2}{|c|}{ Cuestionario final } \\
\hline & Items & $\alpha$ & Items & $\alpha$ \\
\hline N utrición & 14 & 0.68 & 9 & 0.61 \\
\hline Actividad física & 4 & 0.53 & 3 & 0.51 \\
\hline Consumo de tabaco & 2 & 0.96 & 2 & 0.96 \\
\hline Consumo de alcohol & 2 & 0.90 & 2 & 0.90 \\
\hline Familia-amigos & 3 & 0.75 & 0 & NA \\
\hline Información sobre diabetes & 3 & 0.65 & 2 & 0.62 \\
\hline Satisfacción laboral & 4 & 0.50 & 0 & NA \\
\hline Emociones & 6 & 0.83 & 3 & 0.73 \\
\hline Adherencia terapéutica & 3 & 0.65 & 4 & 0.73 \\
\hline Total & 41 & 0.85 & 25 & 0.81 \\
\hline
\end{tabular}

$\mathrm{NA}=\mathrm{N} 0$ aplica 
tabaco, consumo de alcohol e información sobre diabetes, así como en la calificación total, en tanto que los hombres obtuvieron mayor calificación en el dominio emociones, con diferencias estadísticamente significativas (cuadro V).

\section{A nálisis por tiempo de evolución de la DM2}

Al dividir a la muestra en dos grupos, según el tiempo de evolución, en igual o menor de cinco años y mayor de cinco años, se observaron mejores calificaciones en este último grupo en los dominios nutrición, consumo de tabaco y consumo de alcohol, así como en la calificación total, con diferencias significativas (cuadro V).

\section{A nálisis por edad}

Los pacientes con 60 años o más de edad, calificaron mejor en nutrición, consumo de tabaco, emociones, adherencia terapéutica y en la calificación total (cuadro V).

\section{Discusión}

La medición del constructo llamado estilo de vida es una tarea difícil debido a las múltiples dimensiones que lo componen y a la dificultad que entraña su medición directa de una manera objetiva.

El cuestionario de autoadministración desarrollado en el presente estudio, al cual llamamos Instrumento para Medir el Estilo de Vida en Diabéticos (IMEVID), pretende cubrir la necesidad de contar con un instrumento específico que permita al personal de salud

\section{Cuadro III \\ Consistencia EXTERnA TEST-RETEST POR DOMINIO Y total, del Instrumento para Medir \\ el Estilo de Vida en Diabéticos. México, 2001-2002}

\begin{tabular}{lcc} 
Dominios & Valor de r* & Valor de $p$ \\
N utrición & 0.76 & 0.01 \\
\hline Actividad física & 0.68 & 0.01 \\
\hline Consumo de tabaco & 0.84 & 0.01 \\
\hline Consumo de alcohol & 0.78 & 0.01 \\
\hline Información sobre diabetes & 0.67 & 0.01 \\
\hline Emociones & 0.72 & 0.01 \\
\hline Adherencia terapéutica & 0.70 & 0.01 \\
\hline Total & 0.84 & 0.01 \\
*Rho de Spearman & & \\
$n=330$ & & \\
\hline
\end{tabular}

medir el estilo de vida de los pacientes con DM2 de una manera rápida, válida y confiable.

La muestra seleccionada para la validación del IMEVID es representativa de los pacientes diabéticos que acuden a su control médico en las Unidades de Medicina Familiar, del Instituto Mexicano del Seguro Social, en las áreas urbana y suburbana de la Ciudad de México. El amplio espectro en la edad, 19 a 80 años, y el tiempo de evolución, 1 mes a 33 años, permitió buscar diferencias en las calificaciones en distintos estratos de estas variables.

En nuestro estudio predominaron las mujeres, pues ellas acuden con mayor frecuencia que los hom-

Cuadro IV

Medianas de las Calificaciones por dominio y total, Según el género, La edad y el tiempo de eVolución Instrumento para Medir el Estilo de Vida en Diabéticos. México, 2001-2002

\begin{tabular}{|c|c|c|c|c|c|c|c|c|}
\hline \multirow[b]{2}{*}{ Dominios } & \multirow[b]{2}{*}{$\begin{array}{l}\text { Puntos } \\
\text { posibles }\end{array}$} & \multirow[b]{2}{*}{$\begin{array}{l}\text { Mediana } \\
\text { muestral }\end{array}$} & \multicolumn{2}{|c|}{$\begin{array}{l}\text { Mediana por } \\
\text { género }\end{array}$} & \multicolumn{2}{|c|}{$\begin{array}{l}\text { M ediana por } \\
\text { edad (años) }\end{array}$} & \multicolumn{2}{|c|}{$\begin{array}{c}\text { Mediana por } \\
\text { años de evolución }\end{array}$} \\
\hline & & & $\begin{array}{l}\text { Mujeres } \\
(n=269)\end{array}$ & $\begin{array}{l}\text { Hombres } \\
(n=120)\end{array}$ & $\begin{array}{c}<<0 \\
(n=238)\end{array}$ & $\begin{array}{c}\geq 60 \\
(n=151)\end{array}$ & $\begin{array}{c}\leq 5 \\
(n=205)\end{array}$ & $\begin{array}{c}>5 \\
(n=184)\end{array}$ \\
\hline Nutrición & 0 a 36 & 24 & 26 & 22 & 24 & 26 & 24 & 26 \\
\hline Actividad física & 0 a 12 & 6 & 6 & 8 & 6 & 6 & 8 & 6 \\
\hline Consumo de tabaco & 0 a 8 & 8 & 8 & 8 & 8 & 8 & 8 & 8 \\
\hline Consumo de alcohol & 0 a 8 & 8 & 8 & 4 & 8 & 8 & 8 & 8 \\
\hline Información sobre diabetes & 0 a 8 & 4 & 6 & 4 & 6 & 4 & 4 & 6 \\
\hline Emociones & 0 a 12 & 6 & 6 & 6 & 6 & 6 & 6 & 6 \\
\hline Adherencia terapéutica & 0 a 16 & 14 & 14 & 14 & 14 & 14 & 14 & 14 \\
\hline Total & 0 a 100 & 68 & 70 & 66 & 68 & 70 & 66 & 70 \\
\hline
\end{tabular}




\section{Cuadro V \\ Diferencias en los rangos medios de las calificaciones del Instrumento para Medir el Estilo de Vida en Diabéticos, según el género, la edad y el tiempo de evolución. México, 2001-2002}

\begin{tabular}{|c|c|c|c|c|c|c|c|c|c|}
\hline \multirow[b]{2}{*}{ Dominios } & \multicolumn{3}{|c|}{ Género } & \multicolumn{3}{|c|}{ Edad (años) } & \multicolumn{3}{|c|}{ Tiempo de evolución (años) } \\
\hline & $\begin{array}{l}\text { M ujeres } \\
(n=269)\end{array}$ & $\begin{array}{l}\text { Hombres } \\
(n=120)\end{array}$ & $\begin{array}{c}\text { Valor de } \\
p\end{array}$ & $\begin{array}{c}c<0 \\
(n=238)\end{array}$ & $\begin{array}{c}\geq 60 \\
(n=151)\end{array}$ & $\begin{array}{c}\text { Valor de } \\
p\end{array}$ & $\begin{array}{c}\leq 5 \\
(n=205)\end{array}$ & $\begin{array}{c}>5 \\
(n=184)\end{array}$ & $\begin{array}{l}\text { Valor de } \\
\qquad p\end{array}$ \\
\hline Nutrición & 208.63 & 165.96 & 0.001 & 181.77 & 217.23 & 0.002 & 176.82 & 215.26 & 0.001 \\
\hline Actividad física & 193.84 & 199.25 & 0.656 & 192.83 & 199.73 & 0.549 & 201.89 & 187.32 & 0.195 \\
\hline Consumo de tabaco & 207.64 & 168.19 & 0.000 & 188.60 & 206.42 & 0.029 & 184.33 & 206.89 & 0.004 \\
\hline Consumo de alcohol & 216.70 & 147.81 & 0.000 & 189.12 & 205.61 & 0.111 & 183.41 & 207.91 & 0.015 \\
\hline Información sobre diabetes & 209.07 & 164.97 & 0.000 & 197.48 & 192.36 & 0.653 & 185.13 & 205.99 & 0.60 \\
\hline Emociones & 182.37 & 225.03 & 0.000 & 184.63 & 212.71 & 0.015 & 187.24 & 203.65 & 0.143 \\
\hline Adherencia terapéutica & 195.04 & 196.53 & 0.901 & 185.13 & 211.92 & 0.019 & 187.95 & 202.86 & 0.180 \\
\hline Total & 205.78 & 172.38 & 0.007 & 181.79 & 217.20 & 0.002 & 179.14 & 212.67 & 0.003 \\
\hline
\end{tabular}

bres para su atención médica, y generalmente muestran una mejor disposición para participar en este tipo de estudios.

El tiempo necesario para la aplicación del IMEVID es corto, y en su versión final estimamos será de aproximadamente 10 minutos, lo cual permitirá su aplicación inclusive en la sala de espera.

La $\alpha$ de Cronbach de 0.81 para la calificación total, mayor que la obtenida por algunos dominios por separado, confirma que el constructo estilo de vida está integrado por diversos componentes. Este valor, aunque menor que el $\alpha$ reportada para el $\operatorname{HPLP}^{12,23,25,26}(0.92$ a 0.94), refleja un buen nivel de consistencia interna pues se encuentra por arriba de 0.70 y no rebasa 0.90 , punto de corte que, de ser rebasado, podría reflejar redundancia en los ítems. ${ }^{19}$ Los valores $\alpha$ para seis de los siete dominios del IMEVID fueron mayores a 0.60, con un máximo de 0.96 en el dominio consumo de tabaco, y son similares a los valores de $\alpha$ para los siete dominios del HPLP (entre 0.64 y 0.90$)^{12,23,25,26}$ pero menores que los del FANTASTIC (entre 0.08 y 0.80 ). ${ }^{14}$ En el IMEVID el dominio actividad física tuvo el valor más bajo, $\alpha=0.51$, pero se dejó como parte del instrumento por su importancia en la validez de contenido.

La consistencia externa de la calificación total, $r=$ 0.84, muestra un buen nivel de correlación test-retest tomando en cuenta que se trata de un instrumento de auto-administración, que los pacientes diabéticos participantes en nuestro estudio tienen, en su mayoría, menos de seis años de escolaridad, y muchos de ellos son personas mayores, en ocasiones con discapacidades físicas secundarias a la DM o a otras enfermedades concomitantes, que pudieran ocasionarles dificultades al momento de contestar el cuestionario. Este valor del coeficiente de correlación test-retest para la calificación total es similar al obtenido por el FANTASTIC, $r$ entre 0.84 y $0.88,13,14,22$ y por el HPLP, $r$ de 0.86 y $0.84,{ }^{23,26}$ con intervalos de tiempo de 2 a 3 semanas entre las aplicaciones. La correlación test-retest para los dominios del IMEVID, $r$ de 0.67 a 0.84 , es similar a la reportada para los dominios del HPLP, $r$ de 0.70 a $0.87,{ }^{23}$ y para los del FANTASTIC, $r$ de 0.60 a $0.87 . .^{14,21}$

Los dominios del IMEVID final explicaron en conjunto $60.4 \%$ de la varianza de la calificación total, valor mayor a la varianza explicada por los seis dominios del HPLP, entre 45.9 y $52.8 \%$. $12,23,26$ Seis dominios tuvieron un solo componente principal que explicó más de $50 \%$ de la varianza de su calificación y solamente el dominio nutrición mostró estar conformado por dos factores. Los factores identificados en el análisis factorial correspondieron conceptualmente con los dominios que fueron contemplados teóricamente como componentes del estilo de vida en el IMEVID. No se han reportado análisis de factores para el FANTASTIC.

La mejor calificación de las mujeres en el dominio nutrición puede estar asociada con exposición repetida a consejería nutricional por el personal de salud, debido a su mayor asistencia a las unidades de atención médica y, por otro lado, al hecho de que ellas preparan los alimentos lo cual les permite decidir el menú con más facilidad que a los hombres. Las mujeres calificaron más alto en los dominios consumo de alcohol y consumo de tabaco (menor consumo) con una diferencia significativa, lo cual puede explicarse porque el consumo de estas sustancias en los hombres tiene mayor aceptación social, sobre todo el de alcohol, ya 
que el de tabaco tiende a ser igual en ambos géneros, sobre todo en las personas con menor edad. La mayor asistencia de las mujeres a las unidades de atención médica les brinda más oportunidades para acceder a la información y capacitación con respecto a la diabetes, lo que explica su mejor calificación también en este dominio. Los hombres calificaron mejor sólo en el dominio emociones, lo cual pudiera explicarse por la expectativa social de que este género debiera tener un mejor control emocional. Estas diferencias contribuyen a la mejor calificación total obtenida por las mujeres respecto a la obtenida por los hombres.

Los sujetos con más de cinco años desde el diagnóstico de la DM2 mostraron mejores calificaciones en los dominios de nutrición, consumo de alcohol y consumo de tabaco, posiblemente por haber estado expuestos durante más tiempo a acciones de consejería individual o grupal durante los años en los que han acudido para su tratamiento médico.

De la misma forma, los sujetos mayores de 60 años de edad calificaron mejor en los dominios nutrición, consumo de tabaco, emociones y adherencia terapéutica, así como en la calificación total. Mejores calificaciones en las personas mayores también han sido reportadas por Walker y colaboradores ${ }^{27}$ en los dominios responsabilidad para la salud y nutrición, así como en la calificación total del HPLP, y por nuestro grupo en los mayores de 60 años de edad para la calificación total del FANTASTIC. ${ }^{13,14}$ Lo anterior pudiera explicarse porque a mayor edad los pacientes presentan más enfermedades o complicaciones que ensombrecen su pronóstico, por lo que parecen estar dispuestos a modificar sus estilos de vida y seguir las indicaciones médicas, con el fin de mejorar su evolución, preservar la función y prolongar la vida.

Una fuente potencial de sesgo en el presente trabajo es que solamente se estudiaron pacientes diabéticos que acuden para recibir atención en Unidades Médicas del Instituto Mexicano del Seguro Social, localizadas en áreas urbanas y suburbanas, sin encuestar a aquellos que siendo derechohabientes no acuden, a los que reciben servicio médico en otras instituciones de seguridad social, a los que acuden a servicios médicos privados y a los que viven en zonas rurales. Nuevos estudios definirán su utilidad en estos grupos de población.

Otro aspecto importante para considerar en este tipo de cuestionarios es la posibilidad de obtener respuestas socialmente aceptables. Sin embargo, el buen nivel de consistencia externa demostrada por el instrumento, similar a las obtenidas por el FANTASTIC y el HPLP, apoya la suposición de que estas limitaciones son superadas por la robustez del instrumento.

Sabemos que el mejor método para medir el estilo de vida pudiera ser la observación directa de las conductas en la vida cotidiana, pero esta tarea es poco factible de realizar por el personal de salud.

Por otra parte, una ventaja potencial en el sentido de que el instrumento sea de autoadministración es que las respuestas obtenidas reflejan patrones de actitud o conducta asumidas como ciertas por los pacientes, lo cual permite plantear estrategias de cambio partiendo de la propia visión de los sujetos.

En resumen, hemos desarrollado el primer instrumento especifico, en lengua española, para medir el estilo de vida en los pacientes con DM2, con validez lógica y de contenido, así como consistencia interna y externa, propiedades psicométricas que lo convierten en una herramienta potencialmente útil, tanto para uso clínico como para fines de investigación.

Ante la carencia de un estándar de oro adecuado con el cual compararlo, será necesario realizar nuevos estudios para determinar si existe asociación entre las calificaciones obtenidas con el IMEVID y algunas mediciones clínicas objetivas en pacientes diabéticos, como son los parámetros somatométricos y metabólicos, con el fin de sustentar su validez de constructo. En esta evaluación se podrían incluir también los dominios relación con la familia-amigos y satisfacción laboral, con el fin de revalorar su eliminación basada en criterios estadísticos y determinar su utilidad para fines clínicos.

\section{Conclusiones}

El IMEVID es el primer instrumento de autoadministración, en idioma español, diseñado para medir el estilo de vida en pacientes ambulatorios con diabetes mellitus tipo 2, que es fácil de contestar por los encuestados y fácil de calificar para el personal de salud. Puede aplicarse inclusive en la sala de espera para obtener de manera rápida y confiable información útil sobre algunos componentes del estilo de vida que pueden ser de importancia para el curso clínico de esta enfermedad, permitiendo identificar conductas de riesgo, aceptadas como ciertas por los pacientes, que pueden ser potencialmente modificables mediante consejería específica o integración de los sujetos que lo requieran a grupos de autoayuda o de intervención específica.

Herramientas clínicas con estas características pueden ayudar a los médicos y a los pacientes a identificar componentes del estilo de vida desfavorables 
para el curso clínico de la diabetes tipo 2 y a decidir, de manera conjunta, maniobras de intervención específica para modificarlos. La aplicación seriada del instrumento pudiera ser de utilidad también para evaluar el efecto de las intervenciones implantadas.

\section{Agradecimientos}

Agradecemos a las trabajadoras sociales de las Unidades de Medicina Familiar 62, 64, 91, 184, 185 y 186, de la Delegación Estado de México Oriente, del Instituto Mexicano del Seguro Social, por su valiosa ayuda para la realización del trabajo de campo.

\section{Referencias}

1. Mann JI.The role of nutritional modifications in the prevention of macrovascular complications of diabetes. Diabetes 1997;46 (Suppl 2): S125-S130.

2.American Diabetes Association. N utrition recommendations and principles for people with diabetes mellitus. Diabetes Care 1998; 21(Suppl 1):S32-S35.

3.American Diabetes Association. Diabetes mellitus and exercise. Diabetes Care 1998;21(Suppl 1):S40-S44.

4. Bloomgarden ZT. 0 besity and diabetes. Diabetes Care 2000;23: 1584-1590.

5.W ei M, Kampert JB, G ibbons LW, Blair SN , Mitchell TL. Alcohol intake and incidence of type 2 diabetes in men. Diabetes $C$ are 2000;23:18-22. 6. Haire JD, G lasgow RE, Tibbs TL. Smoking and diabetes. Diabetes Care 1999;22(11):1887-1898

7. U situpa MI. Early lifestyle intervention in patients with non-insulin dependent diabetes mellitus and impaired glucose tolerance.Ann Med 1996;28:445-449

8. Franz MJ. Lifestyle modifications for diabetes management. Endocrinol Metab C lin N orth Am 1997;26(3):499-510.

9. World Health 0 rganization. Lifestyles and health. Soc Sci Med 1986;22(2):117-124.

10. W ilson DM, C iliska D. Lifestyle assessment: D evelopment and use of the FAN TASTIC Checklist. C an Fam Physician 1984;30:1527-1532.

11. Bliss C. El estilo de vida y el estándar de vida. En: N ussbaum MC, Sen A. La Calidad de Vida. México, D.F.: 1a. ed. Fondo de Cultura Económica, 1996: 534-557.
12.W alker SN, Sechrist KR, Pender NJ.The Health-Promoting Lifestyle Profile: D evelopment and psychometric characteristics. N urs Res 1987;36(2):76-81.

13. López-C armona JM, Rodríguez-Moctezuma R, Munguía-Miranda C, Hernández-Santiago JL, C asas de la Torre E.Validez y fiabilidad del instrumento "FAN TASTIC" para medir el estilo de vida en pacientes mexicanos con hipertensión arterial. Aten Primaria 2000;26(8):542-549. 14. Rodríguez-Moctezuma R, López-C armona JM, Munguía-Miranda C, Hernández-Santiago JL, Martínez-Bermúdez M.Validez y consistencia del instrumento "FAN TASTIC" para medir el estilo de vida en pacientes mexicanos con diabetes mellitus tipo 2. Rev Med IMSS 2003;41(3): 215-224.

15. G uyatt GH,Veldhuyzen-Van Zanten SJ, Feeny D H, Patrick DL. Measuring quality of life in clinical trials: A taxonomy and review. CMA 1989;140:1441-1448.

16. López JF, Rhode LE, Ponce de León RS. Medición del impacto de intervenciones médicas a nivel individual. Rev Invest Clin 1997;49: 379-386.

17. Anderson R, Fitzgerald J,W isdom K, D avis W, Hiss R.A comparison of global versus disease-specific quality-of-life measures in patients with N ID D M. Diabetes Care 1997;20(3):299-305.

18. Feinstein AR.The evaluation of validity. En: Feinstein AR. C linimetrics. $\mathrm{N}$ ew Haven (CT):Yale University Press, 1987.

19. Streiner D L, N orman GR. Health measurement scales. A practical guide to their development and use. $2^{\text {da }}$ ed. N ueva York (N Y): 0 xford University Press, 1995.

20.Argimon-Pallás JM, Jiménez-Villa J. Métodos de investigación clínica y epidemiológica. $2^{\text {da }}$ ed. Barcelona: Harcourt, 1999.

21. Prieto L, Badia X. Cuestionarios de salud: concepto y metodología. Aten Primaria 2001;28:201-209.

22. W ilson DM, C iliska D. Lifestyle assessment:Testing the FAN TASTIC Instrument. Can Fam Physician 1984;30:1863-1866.

23.W alker SN , Kerr MJ, Pender N J, Sechrist KR.A Spanish language version of the Health- Promoting Lifestyle Profile. N urs Res 1990;39(5): 268-273.

24. Hair JF,Anderson RE,Tatham RL, Black W C . A nálisis multivariante. 5a ed. Madrid: Prentice Hall, 1999.

25. Kuster AE, Fong CM. Further psychometric evaluation of the Spanish language Health-Promoting Lifestyle Profile. N urs Res 1993;42(5): 266-269.

26. Huang YH, Chiou CJ. Assessment of the Health-Promoting Lifestyle Profile on reliability and validity. Kaonsiung J Med Sci 1996;12(9): 529-537.

27. W alker SN, Volkan K, Sechrist KR, Pender N J. Health promoting lifestyles of older adults: comparisons with young and middle-aged adults, correlates and patterns.AN S Adv N urs Sci 1988;11(1):76-90. 


\section{Anexo 1 \\ Cuestionario imeVid*}

\section{Instructivo}

Este es un cuestionario diseñado para conocer el estilo de vida de las personas con diabetes tipo 2. Le agradeceremos que lea cuidadosamente las siguientes preguntas y conteste lo que usted considere que refleja mejor su estilo de vida en los últimos tres meses.

Elija una sola opción marcando con una cruz X en el cuadro que contenga la respuesta elegida.

Le suplicamos responder todas las preguntas.

Fecha:

Nombre:

Sexo: $F \quad M$

Edad: años.

\begin{tabular}{|c|c|c|c|}
\hline 1. ¿Con qué frecuencia come verduras? & $\begin{array}{l}\text { Todos los días } \\
\text { de la semana }\end{array}$ & Algunos días & Casi nunca \\
\hline 2. ¿Con qué frecuencia come frutas? & $\begin{array}{l}\text { Todos los días } \\
\text { de la semana }\end{array}$ & Algunos días & Casi nunca \\
\hline 3. ¿C uántas piezas de pan come al día? & 0 a 1 & 2 & 30 más \\
\hline 4. ¿C uántas tortillas come al día? & 0 a 3 & 4 a 6 & 70 más \\
\hline 5. ¿A grega azúcar a sus alimentos o bebidas? & Casi nunca & Algunas veces & Frecuentemente \\
\hline 6. ¿Agrega sal a los alimentos cuando los está comiendo? & Casi nunca & Algunas veces & Casi siempre \\
\hline 7. ¿Come alimentos entre comidas? & Casi nunca & Algunas veces & Frecuentemente \\
\hline 8. ¿Come alimentos fuera de casa? & Casi nunca & Algunas veces & Frecuentemente \\
\hline $\begin{array}{l}\text { 9. ¿Cuando termina de comer la cantidad servida inicialmente, } \\
\text { pide que le sirvan más? }\end{array}$ & Casi nunca & Algunas veces & Casi siempre \\
\hline $\begin{array}{l}\text { 10. ¿Con qué frecuencia hace al menos } 15 \text { minutos de ejercicio? } \\
\text { (C aminar rápido, correr } 0 \text { algún otro) }\end{array}$ & $\begin{array}{l}3 \text { o más veces } \\
\text { por semana }\end{array}$ & $\begin{array}{l}1 \text { a } 2 \text { veces } \\
\text { por semana }\end{array}$ & Casi nunca \\
\hline 11. ¿Se mantiene ocupado fuera de sus actividades habituales de trabajo? & Casi siempre & Algunas veces & Casi nunca \\
\hline 12. ¿Q ué hace con mayor frecuencia en su tiempo libre? & Salir de casa & Trabajos en casa & Ver televisión \\
\hline 13. ¿Fuma? & No fumo & Algunas veces & Fumo a diario \\
\hline 14. ¿Cuántos cigar rillos fuma al día? & $N$ inguno & 1 a 5 & 60 más \\
\hline 15. ¿Bebe alcohol? & N unca & Rara vez & $\begin{array}{l}1 \text { vez o más } \\
\text { por semana }\end{array}$ \\
\hline 16. ¿C uántas bebidas alcohólicas toma en cada ocasión? & N inguna & 1 a 2 & 30 más \\
\hline 17. ¿A cuántas pláticas para personas con diabetes ha asistido? & 40 más & 1 a 3 & Ninguna \\
\hline 18. ¿Trata de obtener información sobre la diabetes? & Casi siempre & Algunas veces & Casi nunca \\
\hline 19. ¿Se enoja con facilidad? & Casi nunca & Algunas veces & Casi siempre \\
\hline 20. ¿Se siente triste? & Casi nunca & Algunas veces & Casi siempre \\
\hline 21. ¿Tiene pensamientos pesimistas sobre su futuro? & Casi nunca & Algunas veces & Casi siempre \\
\hline 22. ¿Hace su máximo esfuerzo para tener controlada su diabetes? & Casi siempre & Algunas veces & Casi nunca \\
\hline 23. ¿Sigue dieta para diabético? & Casi siempre & Algunas veces & Casi nunca \\
\hline 24. ¿O Ivida tomar sus medicamentos para la diabetes 0 aplicarse su insulina? & Casi nunca & Algunas veces & Frecuentemente \\
\hline 25. ¿Sigue las instrucciones médicas que se le indican para su cuidado? & Casi siempre & Algunas veces & Casi nunca \\
\hline & & & Total \\
\hline
\end{tabular}

* Instrumento para Medir el Estilo de Vida en Diabéticos

Gracias por sus respuestas 